\title{
Quantitative Electron Microscopy and the Application by Single Electron Signals
}

\author{
Ryo Ishikawa ${ }^{1}$, Andrew. R. Lupini ${ }^{2}$, Scott D. Findlay ${ }^{3}$, Takashi Taniguchi ${ }^{4}$, and Stephen J. Pennycook ${ }^{4}$ \\ 1. Institute of Engineering Innovation, University of Tokyo, Bunkyo, Tokyo, Japan. \\ 2. Materials Science and Technology Division, Oak Ridge National Laboratory, Oak Ridge, TN, USA. \\ 3. School of Physics, Monash University, Victoria 3800, Australia. \\ 4. Advanced Key Technologies Division, National Institute for Materials Science, Tsukuba, Japan. \\ 5. Department of Materials Science and Engineering, University of Tennessee, Knoxville, TN, USA.
}

Annular dark field (ADF) imaging in scanning transmission electron microscopy (STEM) is an ideal mode to quantitatively interpret images: the ADF intensity monotonically increases as a function of specimen thickness owing to the incoherent nature. ADF detector calibration combined with frozen phonon image simulations has demonstrate excellent agreement between experiment and simulation [1], which is now actively used in quantitative analysis. Recently we also developed our high-accurate quantitative method by using single electron signals, enabling us to measure some basic physical quantity such as the number of atom per the column [2]. Moreover, it becomes possible to identify the three-dimensional location of a single dopant in bulk materials on the basis of intensity comparison between experiment and simulations [3].

To establish high accurate quantitative measurement, it is of critical importance to understand possible sources of the background signals. Electronic noise can be measureable by acquiring images with the electron beam off. When we turn on the electron beam in a vacuum, distinct increment of the signals is observed in background images, suggesting that a few stray or 'accidental' electrons are impinging on the ADF detector. In addition to a single peak related to DC offset, new discrete peaks are observed with a constant interval in background images (beam on). As a systematic analysis, we developed the method to correct background signals and moreover it becomes possible to estimate the signal level of a single electron. Figure 1 shows the histogram of corrected background image, and discrete peaks appear at the integer number of electrons. Therefore it becomes possible to convert the ADF image with the electron number unit. On the basis of this analysis, we measured specimen thickness with high accuracy $( \pm 1$ atom precision for thin samples) and also determined the inelastic mean free path combined with electron energy-loss spectroscopy.

Once we know the number of atoms per the column, we may also determine the other physical quantities such as absolute defocus value and an effective source size. Fig. 2(a) show ADF STEM image of $w$-AlN viewed along the [1120] direction, where the column contains 15 atoms. To compensate the spatial incoherence of the probe, we assume a Gaussian source and the effective source size is estimated to be $0.62 \AA$ through chi-square test along $X-X^{\prime}$, shown in Fig. 2(b). By convolving the source size with simulated image (Fig. 2(c)), the experimental image is well reproduced, as most evident in Z-contrast profile of Fig. 2(d). Using those quantities, it allows us to analyze image shape of the atom and becomes possible to access three-dimensional information such as the location of a single dopant. 


\section{References}

[1] J.M. LeBeau et al, Phys. Rev. Lett. 100 (2008) 206101.

[2] R. Ishikawa et al, Microsc. Microanal. 20 (2014) 99.

[3] R. Ishikawa et al, Nano Lett. 14 (2014) 1903.

[4] R.I. acknowledges support from JSPS Postdoctoral Fellowship for Research Abroad and Prof. Naoyoa Shibata and Prof. Yuichi Ikuhara (University of Tokyo) for helpful discussions. A.R.L. acknowledges support by the U.S. Department of Energy, Basic Energy Sciences, Materials Sciences and Engineering Division. S.D.F. acknowledges support under the Discovery Projects funding scheme of the Australian Research Council. R.I. and T.T. acknowledge support by a Grant-in-Aid for Scientific Research on Innovative Areas "Nano Informatics" (Grant 25106006) from JSPS.

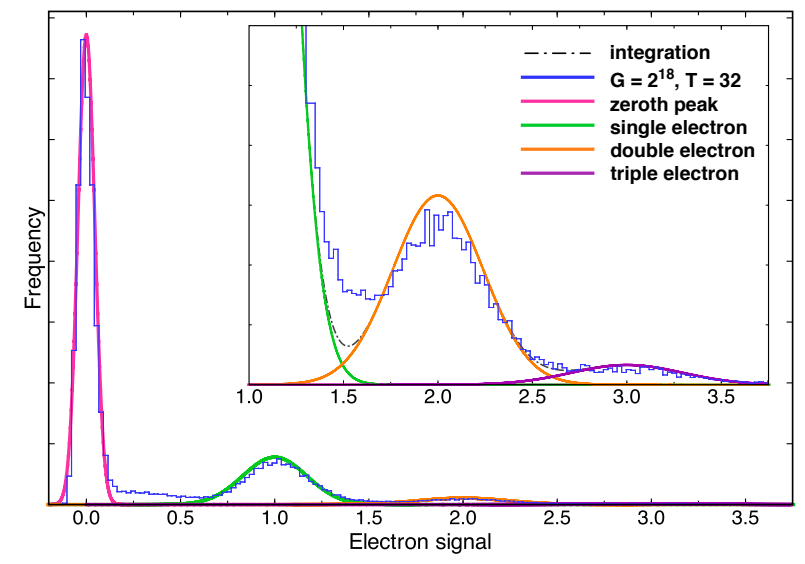

Figure 1. The histogram of the corrected background image, where the horizontal axis is the number of electrons. The overlaid profiles are estimated based on Poisson distribution for the discrete events of single, double and triple electrons.


Figure 2. (a) Atomic-resolution ADF STEM image of $w$-AIN viewed along $a$-axis (15 atom thickness), where the intensity range is $0-2 \%$ of that in the incident electron beam. (b) Simulated ADF STEM image convoluted with a $0.62 \AA$ Gaussian profile. (c) Chi-square test profile as a function of FWHM Gaussian source size. (d) Z-contrast profiles along X-X' for experiment (circles) and simulation (solid line). The scale bar in (a) is $2 \AA$. 